\title{
Investigation of Electrical Properties for Cantilever-Based Piezoelectric Energy Harvester
}

\author{
Ahsan Ali ${ }^{*}$, Riffat Asim Pasha', Muhammad Abdullah Sheeraz ${ }^{2}$, Zubair Butt' ${ }^{2}$, \\ Hassan Elahi ${ }^{3}$, Afzaal Ahmed Khan' \\ 1 Department of Mechanical Engineering, University of Engineering and Technology Taxila, Pakistan \\ ${ }^{2}$ Department of Mechatronics Engineering, University of Engineering and Technology Taxila, Sub Campus \\ Chakwal, Pakistan \\ 3 Department of Mechanical and Aerospace Engineering, La Sapienza University of Rome, Italy \\ * Corresponding author's e-mail: Ahsan.Ali@students.uettaxila.edu.pk
}

\begin{abstract}
In the present era, the renewable sources of energy, e.g., piezoelectric materials are in great demand. They play a vital role in the field of micro-electromechanical systems, e.g., sensors and actuators. The cantilever-based piezoelectric energy harvesters are very popular because of their high performance and utilization. In this researchwork, an energy harvester model based on a cantilever beam with bimorph PZT-5A, having a substrate layer of structural steel, was presented. The proposed energy scavenging system, designed in COMSOL Multiphysics, was applied to analyze the electrical output as a function of excitation frequencies, load resistances and accelerations. Analytical modeling was employed to measure the output voltage and power under pre-defined conditions of acceleration and load resistance. Experimentation was also performed to determine the relationship between independent and output parameters. Energy harvester is capable of producing the maximum power of $1.16 \mathrm{~mW}$ at a resonant frequency of $71 \mathrm{~Hz}$ under $1 \mathrm{~g}$ acceleration, having load resistance of $12 \mathrm{k} \Omega$. It was observed that acceleration and output power are directly proportional to each other. Moreover, the investigation conveys that the experimental results are in good agreement with the numerical results. The maximum error obtained between the experimental and numerical investigation was found to equal $4.3 \%$.
\end{abstract}

Keywords: piezoelectric; micro-electromechanical systems; energy harvesting; microsystems; bimorph

\section{INTRODUCTION}

In the past few decades, there has been an escalation of research in the field of energy harvesting, which is the process being used to obtain the electrical energy from the energy sources available in the environment [1]. Basic renewable sources existing in the ambient environment are: mechanical vibration, wind energy, thermal power, kinetic energy and solar energy; however, with the recent advancement in piezoelectric materials, mechanical vibration is becoming increasingly popular day by day $[2,3]$. Piezoelectric energy harvesters are used as self-powered source in the wireless technology to replace the conventional battery $[4,5]$.
The power generated by a piezoelectric energy harvester can be useful in many applications, such as microelectromechanical systems (MEMS) [6], biomedical sensors [7], piezoelectric miniature robots, and in sensor wireless networks [8]. Piezoelectricity is a property of certain crystalline materials such as PVDF, quartz, ceramics and PZT that develop electricity when mechanical stress is applied. This effect is known as direct piezoelectric effect. Due to the applied electric field, these crystals undergo deformation known as inverse piezoelectric effect. These vibrations can be found in many applications such as moving automobile and structures like bridges and buildings. Human-based energy harvesting 
applications like footstep are also characterized by high amplitude and low frequency displacements $[9,11]$. The obtained electrical energy depends upon several parameters, such as intensity of vibration, expanse of mechanical source present in the environment, sensitivity and performance of electronic devices that are used for power conversion.

Nowadays, the piezo-based harvesters are consistently used in the traffic areas. Although useful-energy is obtained from this source, there is still a problem of low electrical output. In order to eradicate such limitations, a robust energy harvester is observed in [12]. They revealed that the maximum output power of harvester is $3.98 \mathrm{mw}$, which is due to ambient excitation from $2.5 \mathrm{~mm}$ displacement. They also proved that this output is 4.2 times greater than the output of the existing vibrational energy harvesters. On the basis of these results, it is recommended to use this energy harvester in highways to elucidate the problem of energy depletion.

In the past, most of the researchers showed the electrical output of piezo-harvester based on constant ambient excitation. In the case of practical applications, peak-displacement of the cantilever must be limited due to induction of internal strain. Optimization of a piezo-harvester is obtained by considering both maximum displacement and the ambient excitation. Its considered that the efficiency of electromechanical transductions is no more than 50\% [13]. Due to voltage generation of piezoelectric materials, they are massively applied in the field of aerospace industry. The PZT patches are integrated with in the satellite structure to obtain the electrical output due to the application of thermal and outer surface ambient vibrations. The maximum output energy obtained is $0.8 \mathrm{~nJ}$ analyzed by commercially available software ABAQUS with the experimental testing [14]. Alomari et al., studied the electrical output and mode shapes for a unimorph cantilever beam. The effect of single and multiple piezoelectric unimorphs having the same thickness with the change in length and substrate materials on electrical power was examined. Connecting five piezoelectric unimorphs increases the power up to $3 \mu \mathrm{W}$ [15]. There are several techniques to optimize the output response from a energy harvester. One such approach is presented in [16]. It was observed that the maximum power of a bimorph energy harvester exists when the length of piezoelectric layer is taken as $1 / 3$ out of the total length of device. By considering this technique, power density of $2.65 \mathrm{~mW} / \mathrm{cm}^{3}$ was attained. S. Sunithamani studied the effect of proof mass on unimorph piezoelectric harvester and found that using disc proof mass, the maximum output power obtained is $0.443 \mu \mathrm{W}$ [17]. Thickness ratio of substrate to the piezoelectric material can also affect the performance of a piezoelectric energy harvester. S. Sunithamani found that the thickness ratio has a direct relation with frequency [18]. Energy scavenging system based on piezo material, which can harvest energy in all $\mathrm{X}, \mathrm{Y}$ and $\mathrm{Z}$ directions was observed. It was found that seismic mass connected to four L-shape PZT/Si beams has the ability to bend in all three directions. Transverse Piezoelectric mode is used to attain mechanical energy and voltage with durable phase difference generated, depending upon the direction of the applied mechanical vibration. They reveal the fact of attaining $5.53 \mu \mathrm{W}$ in response to $50 \mathrm{mg}$ accelerated vibration [19]. In the past decades, the focus was on the design and development of the techniques for energy scavenging from renewable energy sources. Although piezoelectric materials have a large array of applications in wireless devices, the bandwidth of the harvested energy is too small. The plan of action is based on a device consisting of four piezoelectric fiber composite beams with tip masses and the bandwidth of a harvester is increased by tuning each beam with a different resonance frequency. It was proven that multiple cantilever beams can harvest more energy [20]. During the Piezoelectric transductions based on bimorph cantilever, thickness ratio and electromechanical coupling co-efficient play a vital role to achieve the maximum output power. It was observed that there is an ideal thickness ratio at which the output of bimorph energy harvester reaches maximum. The Young's modulus ratio has inverse relationship with electromechanical coupling coefficient and generated energy [21]. The analysis based on simulation and analytical modeling must have a good agreement to satisfy the output results of energy harvester. In order to eradicate the error existing between them; the approach based on equivalent electrical model is observed. This model is evaluated with resonant frequency and tip deflections. They showed that the model has resonance frequency of $87.12 \mathrm{~Hz}$, satisfying the FEM and analytical results with less than $5 \%$ error [22]. In the past 
few years, several researchers were taking keen interest to design and develop the electronic circuits, which can be used to increase the level of electrical output. A Cuk DC-DC converter is one of them, used to harvest and enhance the electrical energy from various levels of input excitation. Duty cycle plays a typical role during the storage phase of piezo-transductions and it has inverse relationship with the level of ambient vibration. Therefore, a Cuk converter is suggested to raise the efficiency of a piezoelectric energy harvester particularly in which all harvested energy is being absorbed by the conventional battery [23].

In the previous research, many researchers concluded that the phenomenon of energy harvesting via PZT has maximum performance at flutter or resonant frequency $[24,26]$. The aim of this research is to investigate the electrical properties i.e., frequency shocking, to enhance the performance of energy harvester that can have numerous applications in the field of aerospace and mechanical engineering. This research work presents a cantilever model of bimorph piezoelectric energy harvester, which is used to study the effect of excitation frequencies, load resistances and accelerations on output voltage and power. An energy harvester having two layers of piezoelectric material (PZT) and one layer of substrate material was designed and simulated in COMSOL Multiphysics was applied for analyzing the electrical parameters. For validation, experimentation was performed and the experimental results were compared with the numerical results.

\section{THEORETICAL ANALYSIS}

The proposed design of energy harvester is shown in Figure 1. It is based on a cantilever beam having bi-morph piezo-material. Beam under consideration consists of uniform thickness. The central layer is actually sandwiched between the two piezo-electric layers. The material used for the central layer is structural steel. Due to the piezo-materials used on top and bottom surfaces of the cantilever beam, electrodes are defined and these electrodes can be extended for the parallel and series combination in order to enhance the concept of energy harvesting. For the series connection, beams are poled in the opposite direction and then on the application of the force $\mathrm{F}_{\mathrm{s}}$, output voltage is generated on the outer electrodes. For the parallel combination, beams must be polled in the same direction and when force $F_{p}$ is applied, output voltage can be attained by connecting the outer and the center electrodes.

Figure 1 shows that the left-end of this beam is fixed and proof-mass is displaced at the end of its right-end. Due to the end displacement of the mass, bending of a beam takes place, during which two equal and opposite forces act simultaneously on the upper and lower surfaces of the beam. However, there is an axis in the middle of a beam having no change in its length and is known as neutral axis. This research work was done by considering the input vibration or polarization only in the Z-direction and piezo-electric layer experiences strain in the X-direction. For the linear polarization behavior, Piezo constitutive laws in reduced form are given in Equations (1) and (2).

$$
\begin{aligned}
& \mathrm{T}=C_{11}{ }^{E} S+e_{31} E_{3} \\
& \mathrm{D}_{3}=\mathrm{d}_{31} \mathrm{~T}_{1}+\varepsilon_{33}^{S} \mathrm{E}_{3}
\end{aligned}
$$

Sushanta Kundu and Harshal B. Nemade developed analytical modeling based on the bimorph piezo-harvester [27]. The stress developed

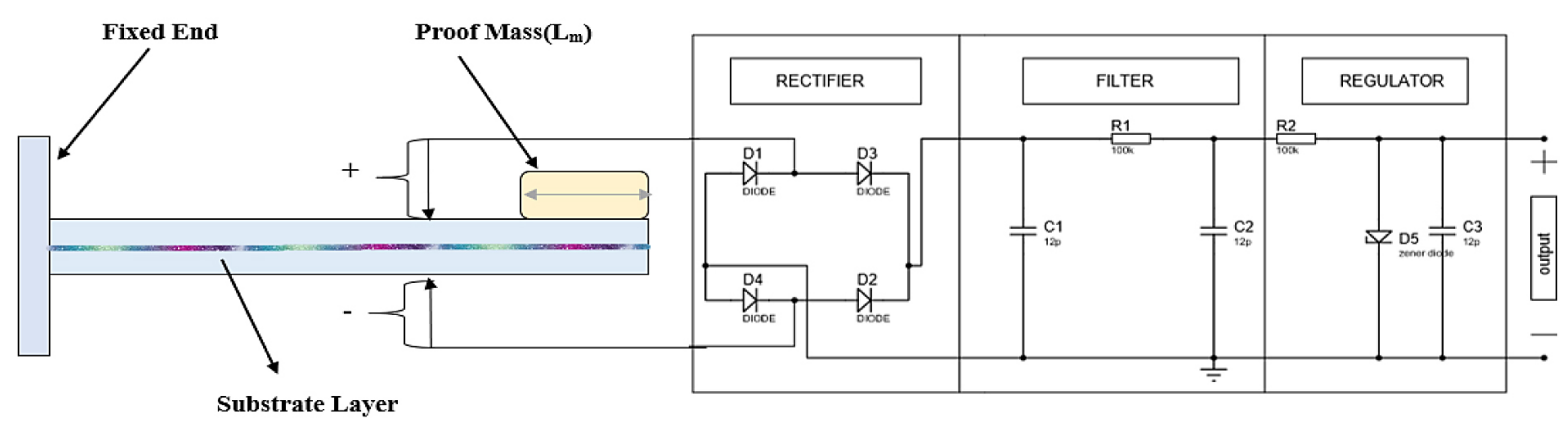

Fig. 1. Bimorph piezoelectric energy harvester 
during the input excitation can be obtained from beam's formula by considering the second moment of area of the cantilever beam, as observed from Equation (3).

$$
\mathrm{T}=\frac{\mathrm{M}}{\mathrm{I}} \times \mathrm{r}_{\mathrm{pc}}
$$

where: $r_{p c}$ is the distance between center of piezolayer and neutral axis.

In order to completely analyze the proposed design, consider the commonly used mass-spring damper system of a vibration energy harvester as shown in the Figure 2. The system consists of the equivalent mass $\mathrm{m}$, spring with spring constant $\mathrm{k}$ and two dampers; Mechanical damper is denoted by $f_{v}$ and $\theta_{E}$ represents electrically induced damping co-efficient.

As tensile and compressive forces are acting on the beam, couple is obtained, the moment of which must be equal to the external moment M, as anticipated from Equation (4).

$$
\mathrm{M}=\mathrm{kz}_{0} \times\left(\mathrm{L}+\left(\frac{L_{m}}{2}\right)-x\right)
$$

In Equation (4), it is observed that strain is induced in $\mathrm{x}$-direction and end-moment of the mass is $\mathrm{kz}_{0}$. Hence, Equation (3) can be rewritten as:

$$
\mathrm{T}=\frac{\mathrm{kz}_{0}\left(\mathrm{~L}+\left(\frac{L_{m}}{2}\right)-x\right)}{I} \times r_{p c}
$$

By considering the electric field as voltage per unit of the piezo-layer $\left(\mathrm{V} / \mathrm{r}_{\mathrm{p}}\right)$ and Equation (5), direct piezo-constitutive equation is given in Equation (6). It must be noted that the stress in Equation (6) is average the stress analyzed from fixed to free end.

$$
\mathrm{D}_{3}=\mathrm{d}_{31}\left(\frac{\mathrm{kz}_{0}\left(\mathrm{~L}+L_{m}\right)}{2 I} \times r_{p c}\right)-\varepsilon_{33}^{S} \times \frac{V}{r_{p}}
$$

Due to alternative distributions, charges will be separated out depending upon the length (L) and thickness (b) of the beam, as obtained from Equation (7).

$$
\mathrm{q}=\mathrm{d}_{31}\left(\frac{\mathrm{kz}_{0}\left(\mathrm{~L}+L_{m}\right)}{2 I} \times r_{p c}\right) b L-\varepsilon_{33}^{S} \times \frac{V}{r_{p}}(b L)
$$

By taking the time-derivative of Equation (7), Current is given in Equation (8)

$$
\mathrm{I}=\mathrm{d}_{31}\left(\frac{K b L\left(L+L_{m}\right) \times r_{p c}}{2 I}\right) \frac{d_{\mathrm{z}_{0}}}{d_{t}}-\frac{\varepsilon_{33}^{S} b L}{r_{p c}} \frac{d_{v}}{d_{t}}
$$

Due to input ambient vibration, alternative voltage is obtained which is further interfaced with the rectifying circuit to obtain the pulsating DC output, as shown in Figure 1. Therefore, Equation (9) represents the electromechanically coupling form of energy harvester

$$
\frac{V}{R}+C_{p} \frac{d_{\mathrm{v}}}{d_{t}}=\theta \frac{d_{\mathrm{z}_{0}}}{d_{t}}
$$

where: $C_{p}$ is Capacitance of a single piezo layer, $\theta$ is electrically induced damping coefficient and V is the Output Voltage.

In this research work, we have connected the piezo-layers in series combination; thus, following the capacitance theory, equivalent capacitance of the energy harvester will be halved with corresponding electromechanically coupling equation given in Equation (10).

$$
2 \frac{V}{R}+C_{p} \frac{d_{\mathrm{v}}}{d_{t}}=2 \theta \frac{d_{\mathrm{z}_{0}}}{d_{t}}
$$

\section{NUMERICAL ANALYSIS OF PZT-5A BIMORPH ENERGY HARVESTER}

The commercially available software COMSOL Multiphysics was used to design and simulate the bimorph piezoelectric energy harvester. Figure 3 shows a geometric model of cantilever based

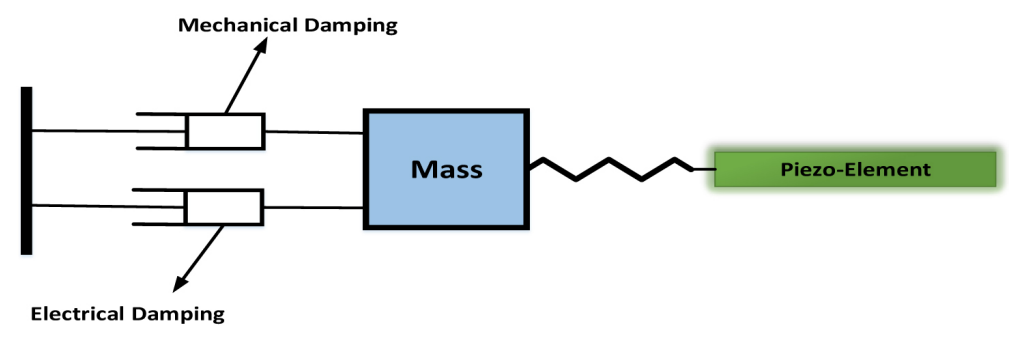

Fig. 2. Mass-spring damper system for energy harvester 
bi-morph energy harvester. PZT-5A and structural steel were used as piezoelectric and substrate materials, respectively, with their properties mentioned in Table 1. The entire geometry of an energy harvester is meshed with free triangular elements. For mechanical boundary conditions, bimorph energy harvester is fixed from its one end. In turn, proof mass having length of $4 \mathrm{~mm}$ and width of $1.5 \mathrm{~mm}$ is placed on its free end to transform the ambient vibration. Overall cantilever beam dimensions are $20 \mathrm{~mm}$ in length and $1.6 \mathrm{~mm}$ in width. Initially, a cantilever is simulated to obtain resonant frequencies by varying the length of proof mass. Afterwards, Bimorph piezoelectric energy harvester is analyzed to obtain output voltage and power in response to several exciting frequencies, load resistances and gravitational acceleration.

\section{EXPERIMENTAL SETUP}

Experimental Testing is performed for validation of numerical results. Figure 4 shows a schematic view of the experimental setup for measuring output voltage and power. The experimental setup shown in Figure 5 consists of Programmable Function Generator (HM8150), Modal Shaker (JZK-10), Power Amplifier (YE5871A), Digital Oscilloscope and Micro Strain WSDA-Base Station. The fixed end of cantilever-based bimorph energy harvester was actually clamped on Modal Shaker acting as a source of vibration and Function generator was used to provide the necessary excitation. Power Amplifier was utilized to control the vibrational frequency of Modal Shaker. Micro strain WSDA-Base station was used to measure the acceleration signal. The output voltage was recorded as a function of excitation frequencies, Load Resistance and acceleration. Power dissipation formula was used to attain the power value across a certain value of load resistance.

\section{RESULTS AND DISCUSSION}

The mechanical and electrical properties of cantilever-based bimorph piezoelectric energy harvester were recorded experimentally as well as numerically. In this section, the obtained experimental and numerical results were discussed and good agreement was observed.

\section{Phase 1: Measurement of resonant frequency}

Resonant frequencies were measured by varying the length of proof mass at free end of cantilever beam. Figure 6 shows the resonant frequencies with various mass lengths having the constant width of proof mass. It was observed that mass length and resonant frequency are inversely proportional to each other. Figure 6 shows that the resonant frequency was decreased from $114 \mathrm{~Hz}$ to $66 \mathrm{~Hz}$ with the variation in length of proof mass ranging from $1 \mathrm{~mm}$ to $5 \mathrm{~mm}$. Due to an increase in the length of proof mass, effective spring constant is reduced which has a direct

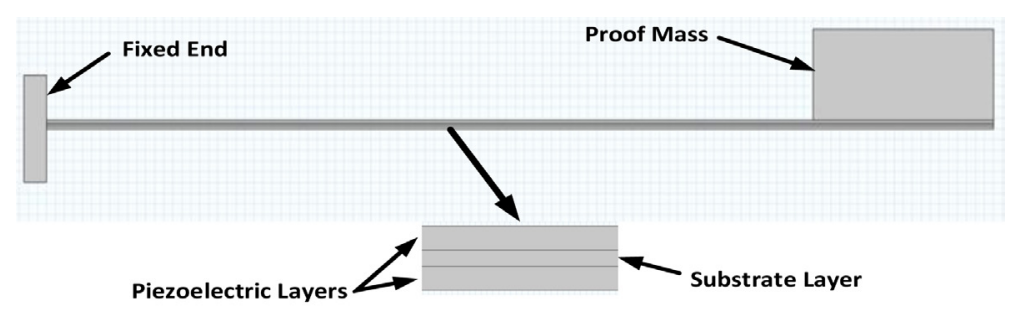

Fig. 3. Cantilever-based bimorph piezoelectric energy harvester

Table 1. Material properties used in simulation [25]

\begin{tabular}{|l|c|c|}
\hline \multicolumn{1}{|c|}{ Parameters } & Symbols & Values \\
\hline Density of piezoelectric material $\left(\mathrm{kgm}^{-3}\right)$ & $\rho_{\mathrm{p}}$ & 7750 \\
\hline Mechanical Quality Factor of piezoelectric material & $\mathrm{Q}_{\mathrm{m}}$ & 75 \\
\hline Young Modulus of piezoelectric material $(\mathrm{Gpa})$ & $\mathrm{E}_{\mathrm{p}}$ & 66 \\
\hline Compliance matrix components $\left(\mathrm{pm}^{2} \mathrm{~N}^{-1}\right)$ & $\mathrm{S}_{11}, \mathrm{~S}_{12}, \mathrm{~S}_{13}, \mathrm{~S}_{33}, \mathrm{~S}_{44}, \mathrm{~S}_{66}$ & $16.4,-5.74,-7.22,18.8,47.5,44.3$ \\
\hline Piezoelectric coupling coefficients $\left(p \mathrm{CN}^{-1}\right)$ & $\mathrm{d}_{31}, \mathrm{~d}_{33}, \mathrm{~d}_{15}$ & $-171,374,584$ \\
\hline Density of substrate material $\left(\mathrm{kgm}^{-3}\right)$ & $\rho_{\mathrm{s}}$ & 7850 \\
\hline Young modulus of substrate material $(\mathrm{Gpa})$ & $\mathrm{E}_{\mathrm{s}}$ & 200 \\
\hline
\end{tabular}


relation with bending modulus per unit width. It reveals the fact that by increasing the proof mass length the bending modulus per unit width will decrease. Consequently, an increase in the length of proof mass will decrease the resonant frequency of cantilever beam. This result shows that variation in proof mass length is a useful parameter for controlling the resonant frequency of energy harvester based on a cantilever beam.

\section{Phase 2: Measurement of voltage and power by varying frequency}

In this phase, voltage and electrical power are evaluated by varying frequency ranging from 50 $\mathrm{Hz}$ to $90 \mathrm{~Hz}$ at an acceleration of $1 \mathrm{~g}$ having load resistance of $12 \mathrm{k} \Omega$. Figure 7 shows that the resonant frequency of cantilever-based bimorph piezoelectric energy harvester is about $71 \mathrm{~Hz}$. At resonant

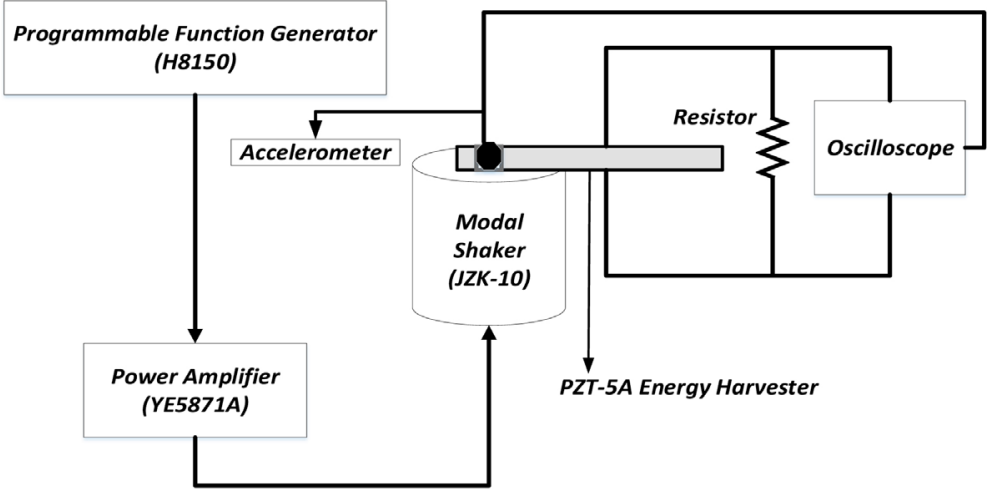

Figure 4. Schematic of energy harvester

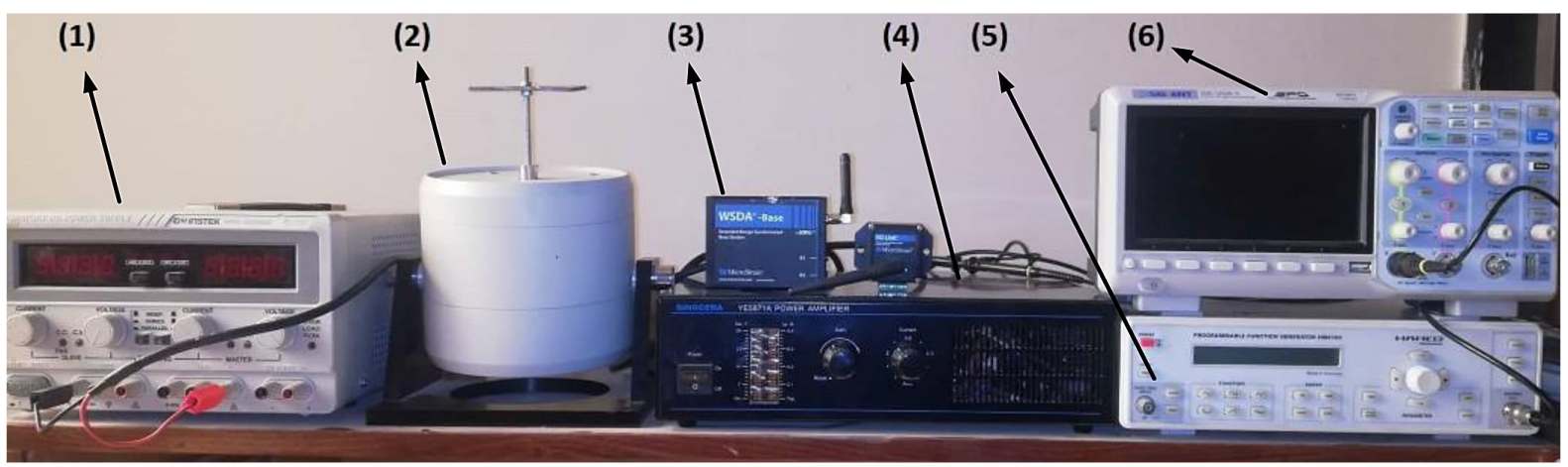

(1)Power Supply (2)Modal shaker JZK-10 (3)Micro Strain WSDA-Base Station (4) Power Amplifier YE5871A

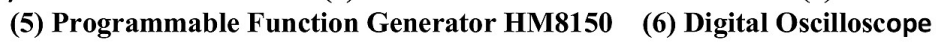

Figure 5. Measurement setup

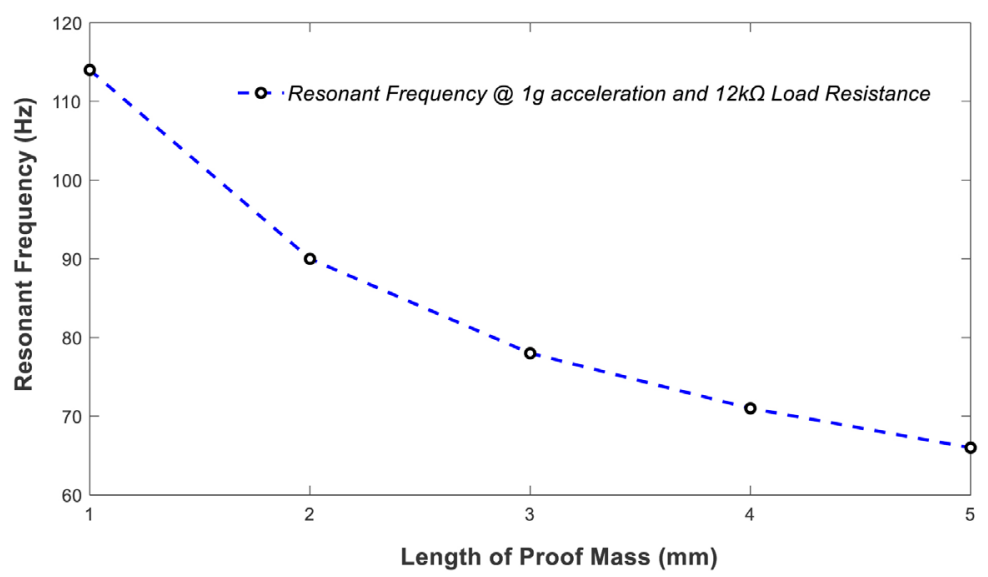

Fig. 6. Measurement of resonant frequency at various lengths of proof mass 
frequency, the maximum value of peak voltage is $5.28 \mathrm{~V}$. Figure 8 shows that at resonant frequency, maximum output power is about $1.16 \mathrm{~mW}$.

\section{Phase 3: Measurement of voltage and power by varying load resistance}

In this phase, voltage and electrical power are analyzed by varying load resistance ranging from $10 \Omega$ to $100 \mathrm{k} \Omega$ at an acceleration of $1 \mathrm{~g}$ having resonant frequency of $71 \mathrm{~Hz}$. Figure 9 and Figure 10 reveal the output voltage and electrical power as a function of load resistance. The output voltage is increased until load resistance becomes $12 \mathrm{k} \Omega$. After $12 \mathrm{k} \Omega$, increasing load resistance does not increase the output voltage significantly. Maximum output power of $1.23 \mathrm{~mW}$ is obtained at $12 \mathrm{k} \Omega$ known as optimal load resistance.

\section{Phase 4: Measurement of voltage and power by varying acceleration}

In this phase, the output voltage and power are measured by varying gravitational acceleration ranging from $0.1 \mathrm{~g}$ to $3.85 \mathrm{~g}$ at an optimal resistance of $12 \mathrm{k} \Omega$. This phase is evaluated at various frequencies from $69 \mathrm{~Hz}$ to $73 \mathrm{~Hz}$. Figures 11 and 12 show the output voltage and power as a function of gravitational acceleration. The results indicate that acceleration and voltage are directly proportional to each other such that by increasing acceleration, output voltage will increase. The maximum output power of $17.24 \mathrm{~mW}$ is obtained at resonant frequency of $71 \mathrm{~Hz}$ having an acceleration of $3.85 \mathrm{~g}$.

\section{Phase 5: Validity of bi-morph energy harvester}

In this phase, the numerical predicted results are compared with the experimental results and an appropriate acceptance is observed. In experimentation, resonant frequency is measured $70.5 \mathrm{~Hz}$ quite nearly equal to $71 \mathrm{~Hz}$ observed numerically. During the experimental testing, the maximum output Voltage and Power are measured as $4.98 \mathrm{~V}$ and $1.11 \mathrm{~mW}$, respectively. Figures 13 and 14 show the comparison between experimentally and numerically predicted output Voltage and Power.

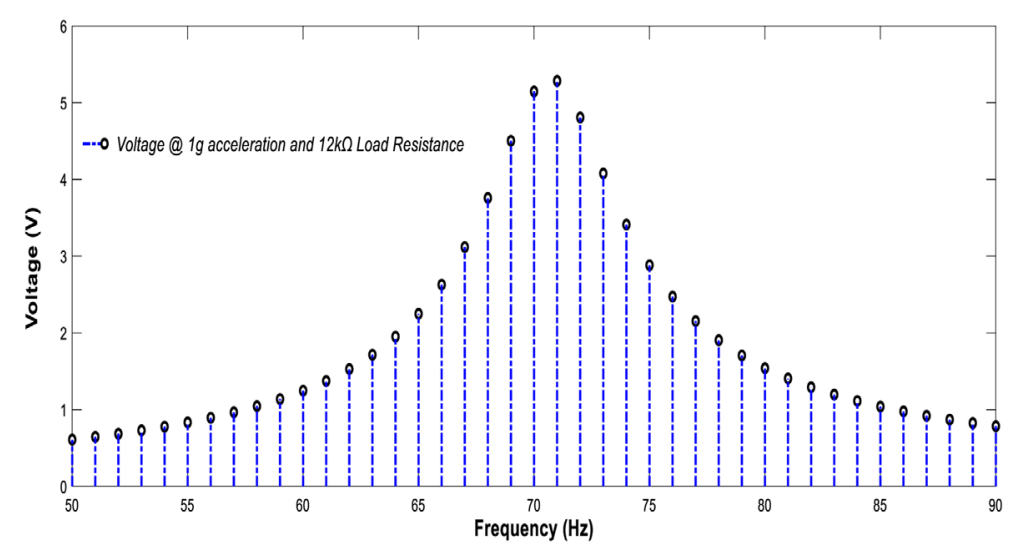

Fig. 7. Measurement of output voltage at various frequencies

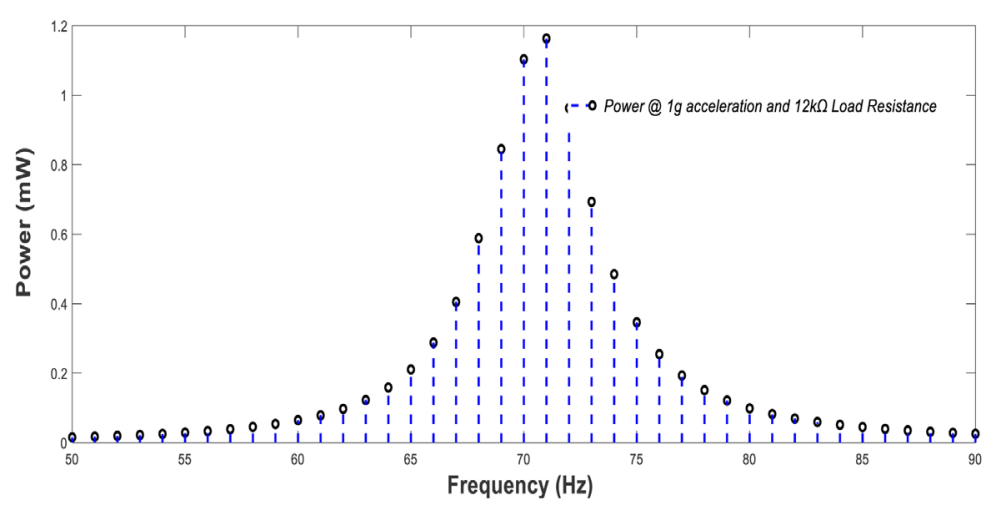

Fig. 8. Measurement of output power at various frequencies 


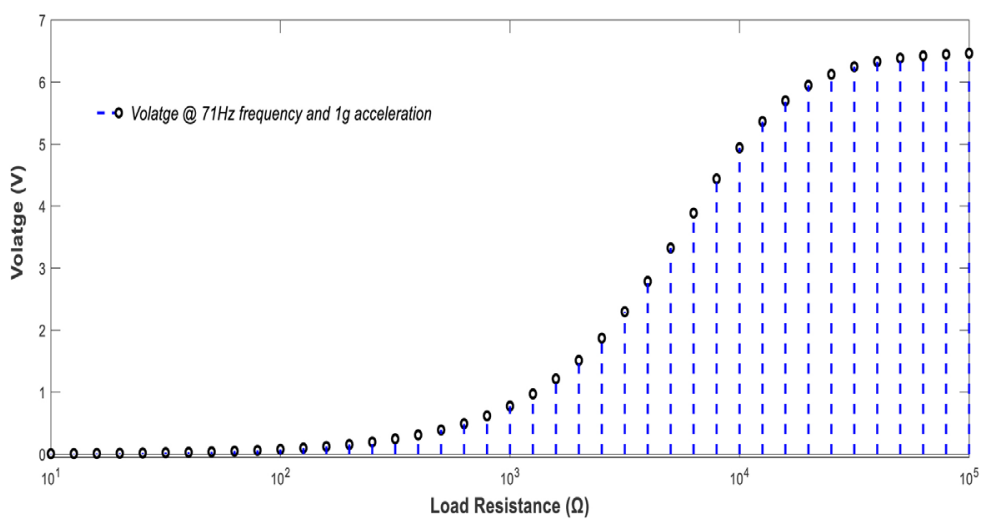

Fig. 9. Measurement of output voltage at various load resistance

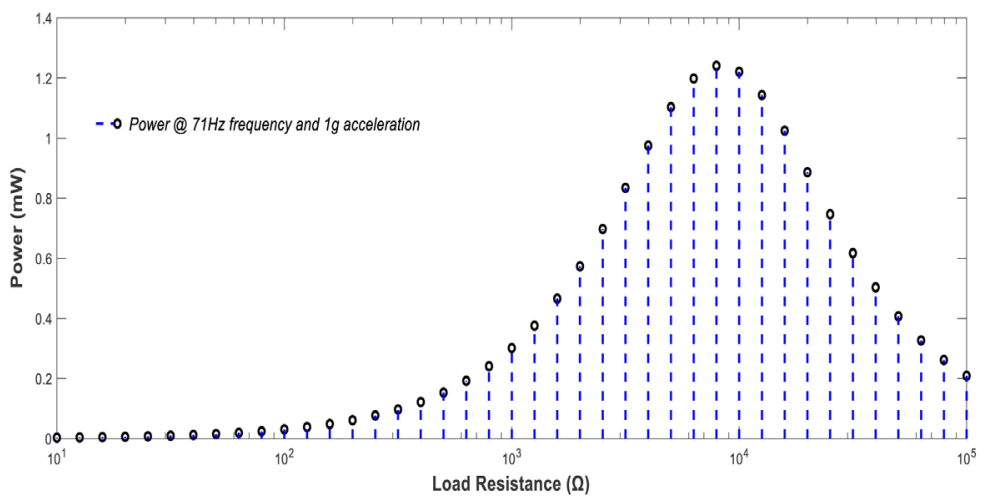

Fig. 10. Measurement of output power at various load resistances

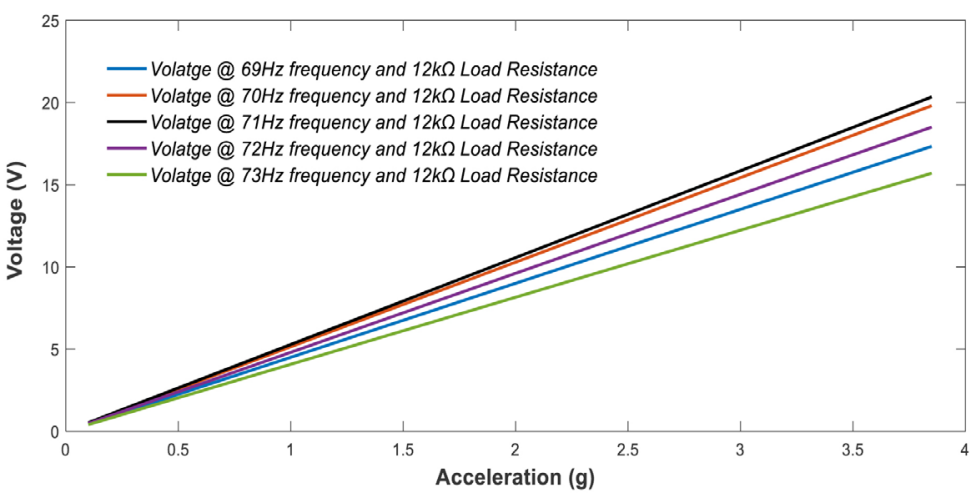

Fig. 11: Measurement of output voltage at various accelerations

\section{CONCLUSION}

In this research paper, a cantilever-based bimorph piezoelectric energy harvester was designed and developed to investigate the direct piezoelectric effect. A numerical analysis was performed by using COMSOL Multiphysics. The performance of piezoelectric energy harvester was investigated at different values of excitation frequency, load resistance and acceleration. The optimal load resistance and resonant frequency of proposed piezoelectric energy harvester are about $12 \mathrm{k} \Omega$ and $71 \mathrm{~Hz}$, respectively. The numerical calculations reveal that the optimal value of resistance is obtained for a particular length of PZT beam and it must be on location that will give maximum value of output power. It was observed that the length of proof mass has an inverse relationship with the resonant frequency of energy harvester. Consequently, an increase in the length 


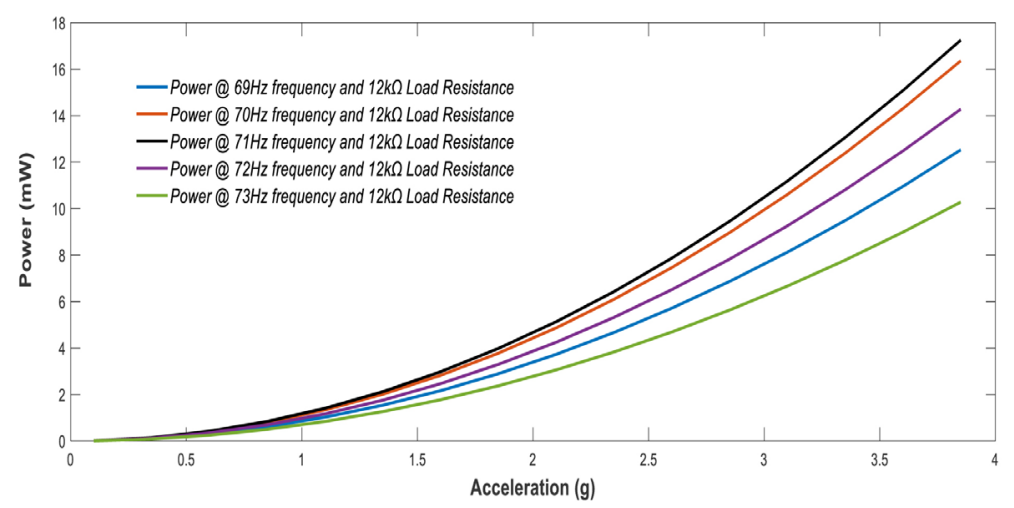

Fig. 12. Measurement of output power at various accelerations

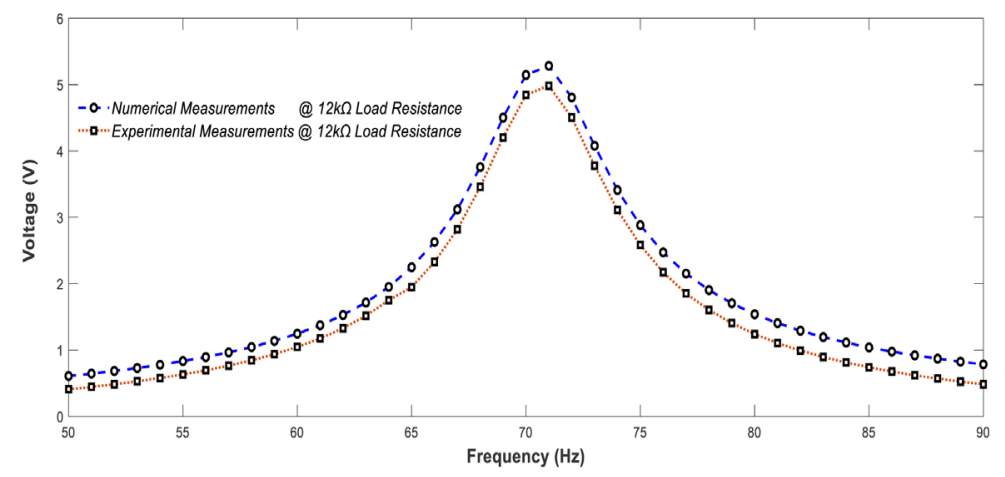

Fig. 13. Measurement of output voltage at $12 \mathrm{k} \Omega$ load resistance and various frequencies

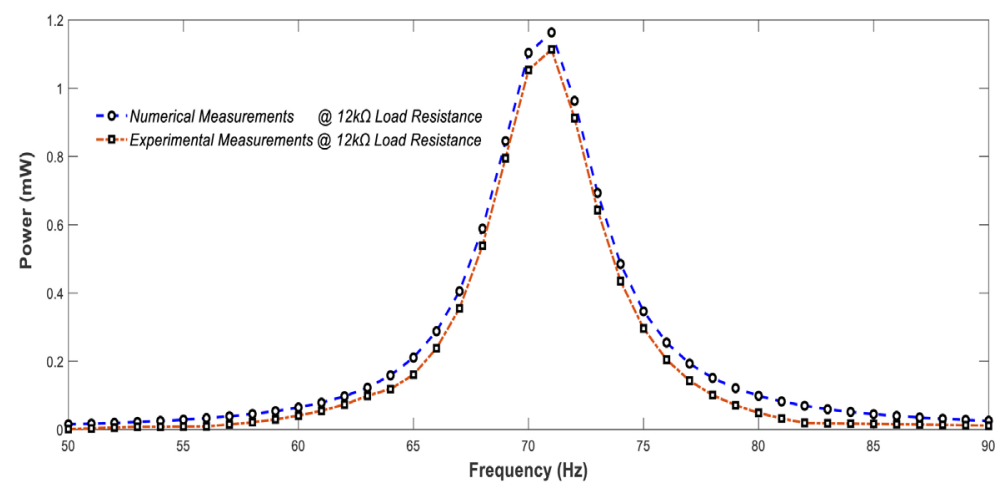

Fig. 14. Measurement of output power at $12 \mathrm{k} \Omega$ load resistance and various frequencies

of proof mass will also increase the output voltage and power. In addition, an energy harvester shows positive linear behavior towards different conditions of acceleration. The experimental testing was performed to verify the numerical predicted results of energy harvester. Energy harvester is capable of producing maximum power of $1.16 \mathrm{~mW}$ at resonant frequency of $71 \mathrm{~Hz}, 1 \mathrm{~g}$ acceleration having $12 \mathrm{k} \Omega$ load resistance. These results enunciate that proposed model is robust and can be connected to an assortment of mechanical conditions. In future work, the system could be improved by considering the effects of damping and precise location of piezo layers on the respective beam.

\section{REFERENCES}

1. Erturk A. and D.J. Inman, An experimentally validated bimorph cantilever model for piezoelectric energy harvesting from base excitations, Smart Mater. Struct., 18(2), 2009, 025009,

2. Priya S., Criterion for material selection in design of bulk piezoelectric energy harvesters. IEEE Trans. Ultrason. Ferroelectr. Freq. Control, 57(12), 2010, 2610-2612. 
3. Pigache F. and C. Nadal, Multimodal electromechanical model of piezoelectric transformers by Hamilton's principle, IEEE Trans. Ultrason. Ferroelectr. Freq. Control, 56(11), 2009, 2530-2543.

4. Poulin G., E. Sarraute, and F. Costa, Generation of electrical energy for portable devices: Comparative study of an electromagnetic and a piezoelectric system, Sensors Actuators, A Phys., 116(3), 2004, 461-471.

5. Priya S. and D.J. Inman, Energy harvesting technologies, Energy Harvest. Technol., 2009, 1-517.

6. Wang Z.L., Nanogenerators for self-powering nanosystems and piezotronics for smart MEMS/ NEMS, Proc. IEEE Int. Conf. Micro Electro Mech. Syst., 2011, 115-120.

7. Roundy S., P.K. Wright, and J. Rabaey, A study of low level vibrations as a power source for wireless sensor nodes, Comput. Commun., 26(11), 2003, 1131-1144,

8. Ottman G.K., H.F. Hofmann, A.C. Bhatt, and G.A. Lesieutre, Adaptive piezoelectric energy harvesting circuit for wireless remote power supply, IEEE Trans. Power Electron., 17(5), 2002, 669-676.

9. Kim H.S., J.H. Kim, and J. Kim, A review of piezoelectric energy harvesting based on vibration, Int. J. Precis. Eng. Manuf., 12(6), 2011, 1129-1141.

10. Hillyard D.C., J. Thompson, A. Kosinski, and P. Mcnabb, Development of an Energy-Harvesting Shoe, University of Tennessee, Knoxville, 2014.

11. Starner T. and J.A. Paradiso, Human-Generated Power for Mobile Electronics, vol. 1990, 2004, 35-45.

12. Ahn J. et al., A Bending-Type Piezoelectric Energy Harvester with a Displacement-Amplifying Mechanism for Smart Highways, vol. 73, 2018.

13. Deng L., Q. Wen, S. Jiang, X. Zhao, and Y. She, On the optimization of piezoelectric vibration energy harvester, J. Intell. Mater. Syst. Struct., 26(18), 2015, 2489-2499.

14. Elahi H., M. Eugeni, P. Gaudenzi, M. Gul, and R. Swati, Piezoelectric thermo electromechanical energy harvester for reconnaissance satellite structure, Microsystem Technologies, 35(2), 2018, 665-672.

15. Alomari A. and A. Batra, Experimental and Modelling Study of a Piezoelectric Energy Harvester Unimorph Cantilever Arrays, Sensors \& Transducers, 192(9), 2015, 37-43.

16. Nechibvute A., A. Chawanda, and P. Luhanga, Enhancing power generation of piezoelectric bimorph device through geometrical optimization, Front. Energy, 8(1), 2014, 129-137.

17. Sunithamani S. and P. Lakshmi, Simulation study on performance of MEMS piezoelectric energy harvester with optimized substrate to piezoelectric thickness ratio, Microsyst. Technol., 21(4), 2015, 733-738.

18. Sunithamani S. and P. Lakshmi, Experimental study and analysis of unimorph piezoelectric energy harvester with different substrate thickness and different proof mass shapes, Microsyst. Technol., 23(7), 2017, 2421-2430.

19. Aktakka E.E. and K. Najafi, Three-axis piezoelectric vibration energy harvester, Micro Electro Mechanical Systems, IEEE International Conference on, 2015, 1141-1144.

20. Oyadiji S., S. Qi, and R. Shuttleworth, Development of Multiple Cantilevered Piezo Fibre Composite Beams Vibration Energy Harvester for Wireless Sensors, In: Kiritsis D., Emmanouilidis C., Koronios A., Mathew J. (Eds) Engineering Asset Lifecycle Management. Springer, London, 2010, 697-704.

21. Kan J., K. Tang, H. Zhao, C. Shao, and G. Zhu, Performance analysis of piezoelectric bimorph generator, Front. Mech. Eng. China, 3(2), 2008, 151-157.

22. Choudhary V. and S. Taruna, Equivalent Circuit Modelling for Unimorph and Bimorph Piezoelectric Energy Harvester, Adv. Informatics Comput. Res., vol. 712, 2017, 39-49.

23. Xue H., H. Hu, Y. Hu, and X. Chen, An improved piezoelectric harvester available in scavengingenergy from the operating environment with either weaker or stronger vibration levels, vol. 52. 2009.

24. Elahi H., M. Eugeni, P. Gaudenzi, F. Qayyum, R. Swati, and H. Khan, Response of piezoelectric materials on thermomechanical shocking and electrical shocking for aerospace applications. 2018.

25. Elahi H., M. Eugeni, and P. Gaudenzi, A Review on Mechanisms for Piezoelectric-Based Energy Harvesters, vol. 11. 2018.

26. Elahi H., M. Eugeni, and P. Gaudenzi, Electromechanical Degradation of Piezoelectric Patches. In: Altenbach H., Carrera E., Kulikov G. (Eds) Analysis and Modelling of Advanced Structures and Smart Systems. Advanced Structured Materials, vol 81. Springer, Singapore, 2018, 35-44.

27. Kundu S. and H.B. Nemade, Modeling and Simulation of a Piezoelectric Vibration Energy Harvester. Procedia Eng., vol. 144, 2016, 568-575. 